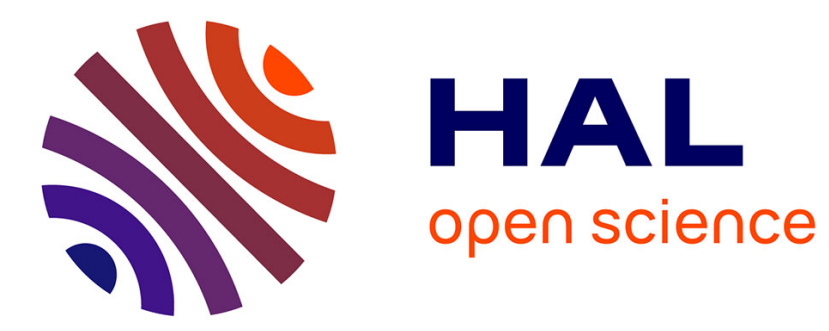

\title{
3D UTD Modeling of a Measured Antenna Disturbed by a Dielectric Circular Cylinder in WBAN Context
}

\author{
Eric Plouhinec, Bernard Uguen, Meriem Mhedhbi, Stéphane Avrillon
}

\section{To cite this version:}

Eric Plouhinec, Bernard Uguen, Meriem Mhedhbi, Stéphane Avrillon. 3D UTD Modeling of a Measured Antenna Disturbed by a Dielectric Circular Cylinder in WBAN Context. IEEE Vehicular Technology Conference (VTC Spring), May 2014, Seoul, South Korea. 10.1109/VTCSpring.2014.7022769 . hal-01122402

\section{HAL Id: hal-01122402 \\ https://hal.science/hal-01122402}

Submitted on 3 Mar 2015

HAL is a multi-disciplinary open access archive for the deposit and dissemination of scientific research documents, whether they are published or not. The documents may come from teaching and research institutions in France or abroad, or from public or private research centers.
L'archive ouverte pluridisciplinaire HAL, est destinée au dépôt et à la diffusion de documents scientifiques de niveau recherche, publiés ou non, émanant des établissements d'enseignement et de recherche français ou étrangers, des laboratoires publics ou privés. 


\title{
3D UTD Modeling of a Measured Antenna disturbed by a Dielectric Circular Cylinder in WBAN Context
}

\author{
Eric Plouhinec \\ LESTP/IETR, \\ Centre de Recherche des Ecoles de Coëtquidan (CREC), \\ 56381 Guer Cedex, France \\ Email: eric.plouhinec@st-cyr.terre-net.defense.gouv.fr \\ Bernard Uguen, Meriem Mhedhbi and Stéphane Avrillon \\ IETR UMR CNRS 6164, \\ Université de Rennes 1, France
}

\begin{abstract}
This paper describes a work realized for On-Body antennas characterization: the 3D deterministic modeling of a measured antenna disturbed by a dielectric circular cylinder of finite length. This prediction model is based on the ray-tracing technique for the electromagnetic wave paths search and the Uniform Theory of Diffraction (UTD) for the modeling of the electromagnetic waves interactions with the cylinder. After a detailed description, the model is validated in 3D with measurements made for an antenna disturbed by a cylindrical phantom [1]. Indeed, the presented model gives results very close to these measurements. These first validations allow the presented model to be implemented into more complex WBAN (Wireless Body Area Networks) propagation models.
\end{abstract}




\section{Introduction}

This paper is related to WBAN (Wireless Body Area Networks) propagation modeling [2] [3]. It is focused on investigating and modeling the interaction of the antenna with the body, according to its orientation and proximity. One possibility is the deterministic WBAN channel modeling using UTD (Uniform Theory of Diffraction). UTD allows the received electromagnetic field computation associated with ray-tracing. This theory is only used with canonical shapes implying that human body could be modeled by cylinders, which is a widely used approximation [4]- [9]. The dielectric cylinder could model, for instance, an arm, the trunk or a leg.

In this paper, a 3D deterministic propagation prediction model is presented, specific to a measured antenna disturbed by a 3D circular dielectric cylinder. The used ray-tracing technique and the UTD formulations are detailed. This model has already been validated in 2D with a conducting and a dielectric cylinder using [10] [11] and [12] respectively. Concerning the 3D approach, it has been validated for a conducting cylinder thanks to [13]. Consequently, in this paper, we focus on a 3D dielectric cylinder approach. The model is compared with measurements made for the characterization of a body-worn antenna relying on a specific OTA (Over-The-Air) test-bed in anechoic chamber and a near-field antenna measurement chamber [1], for various distances between the antenna and a cylindrical phantom.

This document is structured as follows: in Section II, the 3D deterministic model is described: we particularly focus on the specific 3D ray-tracing used for an electromagnetic source in presence of a circular dielectric finite length cylinder and the specific UTD formulations used for the computation of the total received electromagnetic field. Then, in Section III, we describe briefly the measurement campaign. Finally, in Section IV, we focus on the validation of the model, making a 3D comparison between the presented model results and measurements.

\section{3D Deterministic Model Description}

\subsection{Ray-Tracing}

First, we have to look for the different rays existing between a Source Point $S$ and a Receiver Point $P$ in presence of a 3D finite length circular cylinder of 
radius $R$. The cylinder, $S$ and $P$ could be placed anywhere in the cartesian coordinates system but $S$ and $P$ can not be too close to the cylinder because only UTD "Scattering" case [10] is studied in this paper. But, to make the computation easier, the cylinder axis is necessarily brought back to $z$ axis and the $S$ ordinate is brought back to the "null" value. We will call $A$ (resp. $B$ ) the bottom disk center (resp. the top disk center) of the cylinder, as illustrated, for example, by Fig. 1 (a).

So, we could have 2 ray-tracing scenarios: $S$ and $P$ could be in Line Of Sight (LOS) as illustrated by Fig. 1 (a) or in Non Line Of Sight (NLOS) as illustrated by Fig. 1 (b). For a LOS scenario, the ray-tracing finds a maximum of 4 rays, as illustrated by Fig. 1 (a): 1 direct ray (in brown), 1 reflected ray (in blue) with reflection point $Q_{R}$, and 2 diffracted or "creeping"' rays (in red and green). The "red" (resp. "green") ray, of attachment point $Q_{1}$ (resp. $Q_{3}$ ) and detachment point $Q_{2}$ (resp. $Q_{4}$ ) will be called ray 1 (resp. ray 2 ) in the following. By convention, ray 1 (resp. ray 2 ) has its $x y$ plane projection "creeping" in the clockwise (resp. counterclockwise) direction.

For a NLOS scenario, only a maximum of 2 diffracted rays are found by the ray-tracing, as illustrated by Fig. 1 (b). We have to notice that, even if the treated case is canonical, rays diffracted by the bottom and top disks of the cylinder are not taken into account: indeed, they will not appear in WBAN because these disks are not parts of human bodies.

Finding the reflection point $Q_{R}$ in $2 \mathrm{D}$ could not be solved by ruler and compass : for this, we have to find the minimum of the scalar product $\widehat{n} .\left(\frac{\overrightarrow{S Q_{R}}}{\left\|\overrightarrow{S Q_{R}}\right\|}+\frac{\overrightarrow{Q_{R} P}}{\left\|\overrightarrow{Q_{R} P}\right\|}\right)$, where $\widehat{n}$ is the circle normal at $Q_{R}$, using an optimization function. Then, the reflection point height is obtained by an appropriated Thales theorem application. Concerning the attachment and detachment points of the "creeping" part of the diffracted rays, they are easily obtained in 2D using trigonometric formulas [10]. To obtain these points heights, we have to notice that the "creeping" part of the ray is necessarily a cylinder geodesic. If we "unfold" the cylinder, it becomes a plane and this geodesic becomes a straight line: then, these heights are easily found using the Thales theorem in the appropriated planes.

Moreover, we need to verify if reflection, attachment or detachment points are on the cylinder because of its finite length. Finally, to test if $S$ and $P$ are in NLOS, we have to detect if $\overrightarrow{S P}$ is blocked by the $A$ center disk, or the $B$ center disk, or the rectangular cross-section of the cylinder, perpendicular to $x y$ plane and containing $Q_{1}$ and $Q_{3}$ [13] [14]. 


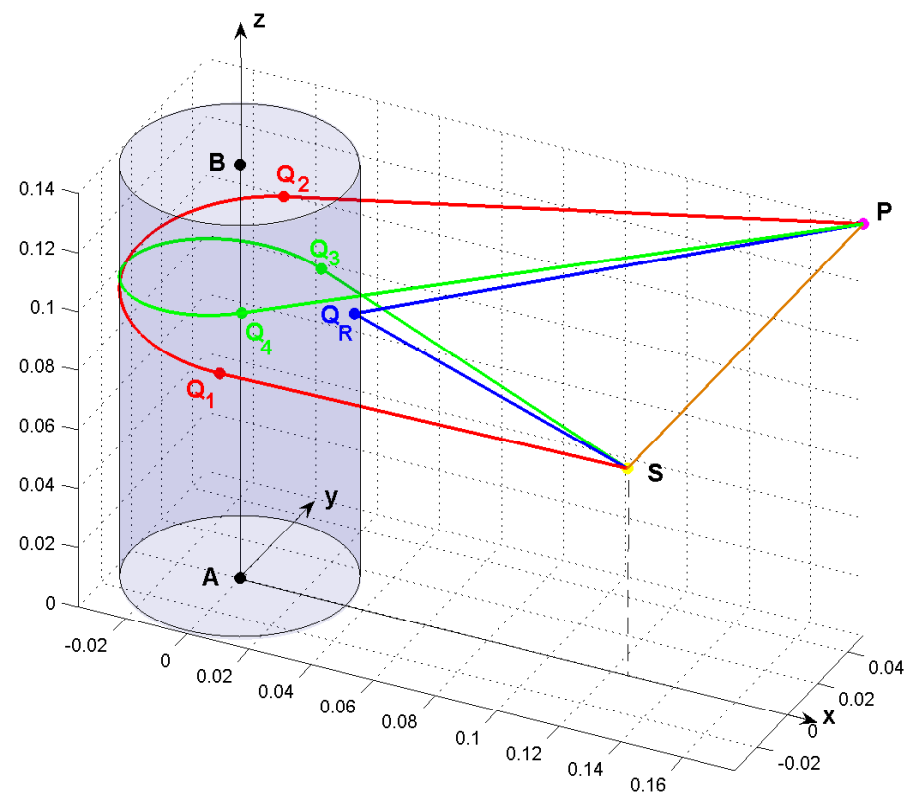

(a) LOS case

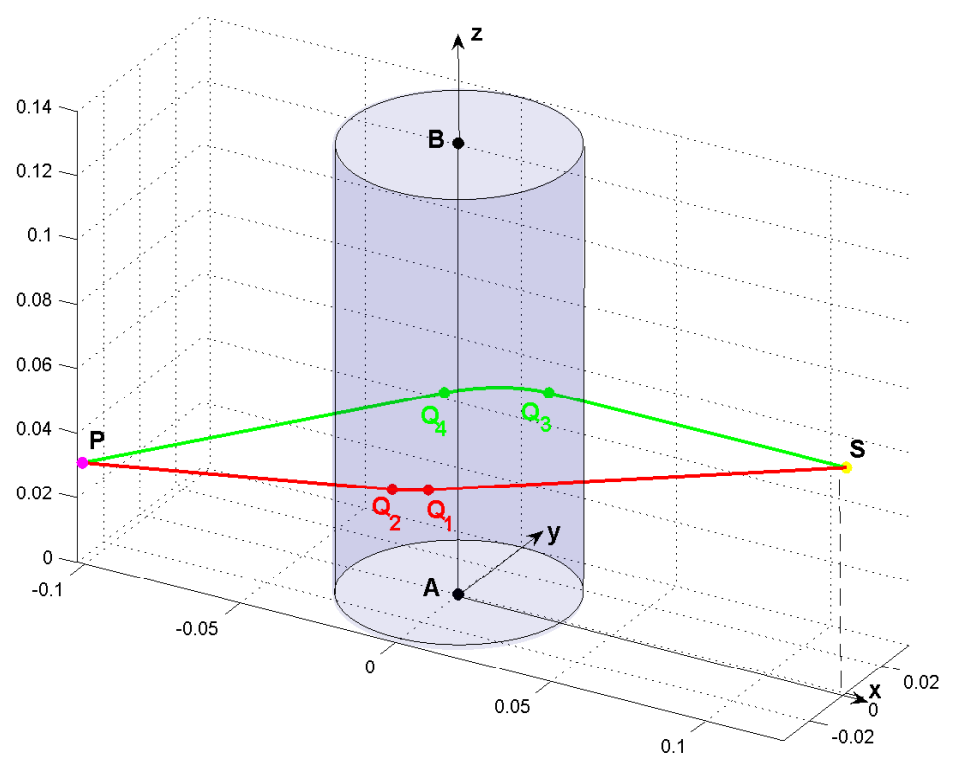

(b) NLOS case

Figure 1: 3D Ray-tracing illustrations 


\subsection{UTD Interactions Modeling}

\subsubsection{Received Total Field Expression}

First, the incident (or direct) field is expressed as:

$$
\mathbf{E}_{\theta, \phi}^{i}(P t)=\mathbf{F}_{e}\left(\theta_{e_{P t}}, \phi_{e_{P t}}\right) \frac{e^{-j k s^{s r c}(P t)}}{s^{s r c}(P t)}
$$

where $s^{s r c}(P t)$ is the distance between $S$ and point $P t, k$ is the well-known wave number and:

$$
\mathbf{F}_{e}\left(\theta_{e_{P t}}, \phi_{e_{P t}}\right)=F_{\theta}\left(\theta_{e_{P t}}, \phi_{e_{P t}}\right) \widehat{\theta}_{e_{P t}}+F_{\phi}\left(\theta_{e_{P t}}, \phi_{e_{P t}}\right) \widehat{\phi}_{e_{P t}}
$$

is the value of the antenna pattern in the $\left(\theta_{e_{P t}}, \phi_{e_{P t}}\right)$ direction from $S$ to Pt.

Secondly, the reflected field is expressed as:

$$
\mathbf{E}_{\theta, \phi}^{r}(P)=A_{r} \mathbf{M}_{2}^{r} \mathbf{R} \mathbf{M}_{1}^{r} \mathbf{E}_{\theta, \phi}^{i}\left(Q_{R}\right) e^{-j k s^{o b s}\left(Q_{R}\right)}
$$

with $\mathbf{R}=\left[\begin{array}{cc}R_{s} & 0 \\ 0 & R_{h}\end{array}\right]$ the dyadic matrix containing the reflection coefficients $R_{s}$ and $R_{h}$ for polarization "soft"(s) and "hard"(h) [10] [11] respectively, $s^{\text {obs }}\left(Q_{R}\right)$ the distance between $Q_{R}$ and $P, A_{r}$ the reflection spreading factor [11], $\mathbf{M}_{1}^{r}$ the $2 \mathrm{D}$ rotation matrix around $\overrightarrow{S Q_{R}}$ from $\left(Q_{R}, \widehat{\theta}_{e_{Q_{R}}}, \widehat{\phi}_{e_{Q_{R}}}\right)$ to $Q_{R}$ local incident base [11] and $\mathbf{M}_{2}^{r}$ the $2 \mathrm{D}$ rotation matrix from $Q_{R}$ local reflected base [11] to $\left(Q_{R}, \widehat{\theta}_{r_{P}}, \widehat{\phi}_{r_{P}}\right)$ with $\left(\theta_{r_{P}}, \phi_{r_{P}}\right)$ the direction from $Q_{R}$ to $P$.

Thirdly, the diffracted field (due to ray 1 or 2 ) is:

$$
\begin{aligned}
\mathbf{E}_{\theta, \phi}^{d 1,2}(P)= & A_{d}^{1,2} \mathbf{M}_{2}^{d 1,2} \mathbf{T}^{1,2} \mathbf{M}_{1}^{d 1,2} \\
& \cdot \sqrt{\frac{d \eta\left(Q_{1,3}\right)}{d \eta\left(Q_{2,4}\right)}} \mathbf{E}_{\theta, \phi}^{i}\left(Q_{1,3}\right) e^{-j k s^{o b s}\left(Q_{2,4}\right)}
\end{aligned}
$$

with $\mathbf{T}^{1,2}=\left[\begin{array}{cc}T_{s}^{1,2} & 0 \\ 0 & T_{h}^{1,2}\end{array}\right]$ the dyadic matrix containing the diffraction coefficients associated with ray 1 or $2, A_{d}^{1,2}$ the diffraction spreading factor [11] associated with ray 1 or $2, \mathbf{M}_{1}^{d 1,2}$ the $2 \mathrm{D}$ rotation matrix around $\overrightarrow{S Q_{1,3}}$ from $\left(Q_{1,3}, \widehat{\theta}_{e_{Q_{1,3}}}, \widehat{\phi}_{e_{Q_{1,3}}}\right)$ to $Q_{1,3}$ local incident base [11] and $\mathbf{M}_{2}^{d 1,2}$ the 2D rotation matrix from $Q_{2,4}$ local diffracted base [11] to $\left(Q_{2,4}, \widehat{\theta}_{d 1,2_{P}}, \widehat{\phi}_{d 1,2_{P}}\right)$ with 
$\left(\theta_{d 1,2_{P}}, \phi_{d 1,2_{P}}\right)$ the direction from $Q_{2,4}$ to $P$. Moreover, the $\sqrt{\frac{d \eta\left(Q_{1,3}\right)}{d \eta\left(Q_{2,4}\right)}}$ factor illustrates the conservation of energy flux in the surface-ray strip from $Q_{1,3}$ to $Q_{2,4}[11]$.

Finally, the total received field is computed thanks to the following expression:

$$
\mathbf{E}_{\theta, \phi}^{T}(P) \simeq \alpha\left(\mathbf{E}_{\theta, \phi}^{i}(P)+\beta_{r} \mathbf{E}_{\theta, \phi}^{r}(P)\right)+\sum_{i=1}^{2} \beta_{d i} \mathbf{E}_{\theta, \phi}^{d i}(P)
$$

$\alpha=1$ (resp. $\alpha=0$ ) in the LOS (resp. NLOS) case. $\beta_{r}=1$ if $Q_{R}$ is on the cylinder, $\beta_{r}=0$ otherwise. $\beta_{d 1}=1$ (resp. $\beta_{d 2}=1$ ) if $Q_{1}$ and $Q_{2}$ (resp. $Q_{3}$ and $Q_{4}$ ) are on the cylinder, $\beta_{d 1}=0$ (resp. $\beta_{d 2}=0$ ) otherwise. We have also to notice that even if $(s, h)$ polarization is generally used for the UTD computation [10] [11], we obtain the received field in the $(\theta, \phi)$ polarization which is directly related to the well-know spherical coordinates system used for the following measurements.

\subsubsection{Interaction Coefficients}

First, we have to define the curvature parameter $m(P t)$ expressed as:

$$
m(P t)=\left(\frac{k R_{c}(P t)}{2}\right)^{\frac{1}{3}}
$$

where $R_{c}(P t)$ is the cylinder radius of curvature along the geodesic containing point $P t$, and the distance parameter $L\left(P t_{1}, P t_{2}\right)$ expressed as:

$$
\left(L\left(P t_{1}, P t_{2}\right)\right)^{-1}=\left(s^{s r c}\left(P t_{1}\right)\right)^{-1}+\left(s^{o b s}\left(P t_{2}\right)\right)^{-1}
$$

We can now define the reflection coefficients as:

$$
R_{s, h}=-\sqrt{\frac{-4}{\xi_{L}}} e^{-j \frac{\left(\xi_{L}\right)^{3}}{12}} G_{s, h}\left(\xi_{L}, X_{L}\right)
$$

with:

$$
\xi_{L}=-2 m\left(Q_{R}\right) \cos \theta^{i} \quad \text { and } \quad X_{L}=2 k L\left(Q_{R}, Q_{R}\right) \cos ^{2} \theta^{i}
$$

with $\theta^{i}$ the reflection angle respect to cylinder normal at $Q_{R}$.

And the diffraction coefficients are expressed as:

$$
T_{s, h}^{1,2}=-m\left(Q_{1,3}\right) \sqrt{\frac{2}{k}} e^{-j k t_{1,2}} G_{s, h}\left(\xi_{d 1,2}, X_{d 1,2}\right)
$$


with $t_{1}$ (resp. $t_{2}$ ) the length of the considered cylinder geodesic between $Q_{1}$ and $Q_{2}$ (resp. $Q_{3}$ and $Q_{4}$ ) and:

$$
\xi_{d 1,2}=\frac{m\left(Q_{1,3}\right)}{R_{c}\left(Q_{1,3}\right)} t_{1,2} \quad \text { and } \quad X_{d 1,2}=\frac{k L\left(Q_{1,3}, Q_{2,4}\right)\left(\xi_{d 1,2}\right)^{2}}{2\left(m\left(Q_{1,3}\right)\right)^{2}}
$$

Finally, a common function $G_{s, h}(\xi, X)$ appears in equations (8) and (10). This function is expressed as:

$$
G_{s, h}(\xi, X)=\frac{e^{-j \pi / 4}}{2 \xi \sqrt{\pi}}[1-F(X)]+\widehat{P}_{s, h}(\xi)
$$

depending on the "Transition" function $F(X)$ expressed as:

$$
F(X)=2 j \sqrt{X} e^{j X} \int_{\sqrt{X}}^{+\infty} e^{-j t^{2}} d t
$$

and on the "Pekeris" function $\widehat{P}_{s, h}(\xi)$ expressed as:

$$
\widehat{P}_{s, h}(\xi)=\frac{e^{-j \pi / 4}}{\sqrt{\pi}} \int_{-\infty}^{+\infty} e^{-j \xi t} \frac{V^{\prime}(t)-q_{s, h} V(t)}{W_{2}^{\prime}(t)-q_{s, h} W_{2}(t)} d t
$$

Fock-type Airy functions $V(t), V^{\prime}(t), W_{2}(t)$ and $W_{2}^{\prime}(t)$ appearing in equation (14) could be expressed according to the well-known Airy functions [15]. The "Pekeris" function is the only term that takes into account the dielectric nature of the cylinder depending on polarization, thanks to $q_{s, h}$ parameter expressed as:

$$
q_{s}=-j m(P t) K \quad \text { and } \quad q_{h}=-j \frac{m(P t)}{K}
$$

with $P t=Q_{R}$ for the reflected ray, $P t=Q_{1}$ (resp. $Q_{3}$ ) for ray 1 (resp. ray 2) and:

$$
K=\sqrt{\epsilon_{r}}=\sqrt{\epsilon_{r}^{\prime}-j\left[\epsilon_{r}^{\prime \prime}+60 \lambda \sigma\right]}
$$

with $\epsilon_{r}$ the complex permittivity of the cylinder and $\lambda$ the wavelength. $\epsilon_{r}^{\prime}, \epsilon_{r}^{\prime \prime}$ and $\sigma$ are the cylinder dielectric properties and are called, respectively, the relative permittivity real part, the relative permittivity imaginary part and the conductivity. 


\section{Measurement Campaign Description}

The measurement campaign was carried out in the Satimo SG32 near field antenna mesurement chamber of IETR ("Electronics and Telecommunications Institute of Rennes") illustrated by Fig. 2 (a). The cylinder under test is a custom built phantom depicted by Fig. 2 (b). It is nearly a cylindrical plastic bottle filled with MSL1800 phantom liquid. The dielectric properties of this liquid are given in [16] for the $[0.3-3] \mathrm{GHz}$ frequency range. The size of the phantom of radius $R=3.5 \mathrm{~cm}$ was chosen to represent a human arm. The bottle was fitted with a screw in the bottom to fix it on the platform grooves or holes (cf. Fig. 3). The used Ultra Wide Band (UWB) printed circular dipole antenna [17] was placed in the centre of the measurement cartesian coordinates system illustrated in Fig. 3. Its azimuth radiation pattern (here in the $x y$ plane) is omnidirectional in the $[3-6] G H z$ frequency range.

The measurements were made in the $[0.8-5.95] \mathrm{GHz}$ frequency range for various antenna-phantom distances $d$ varying from $3 \mathrm{~cm}$ to $13.5 \mathrm{~cm}$ [1]. The measurements were carried out in the spherical coordinates system $(\widehat{r}, \widehat{\theta}, \widehat{\phi})$, for $\theta \in\left[0^{\circ} ; 180^{\circ}\left[\right.\right.$ and $\phi \in\left[0^{\circ} ; 360^{\circ}\left[\right.\right.$ with a $2^{\circ}$ step, for the received farfield as well as for the "antenna without phantom" radiation pattern called $F_{e}\left(\theta_{e_{R x}}, \phi_{e_{R x}}\right)$ (directly included in the presented model thanks to equation (2)) with receiver $R x$ in the far-field region.

\section{Comparison Results}

For this comparison, the cylinder axis is the $z$ axis to be coherent with the measurements cartesian coordinate system. So, for the model, cylinder point $A$ becomes the cartesian coordinates system origin (cf. Fig. 1), different from the measurements system origin (cf. Fig. 3). $S$ has an ordinate $y_{s}=0$ and consequently an abscissa $x_{s}=d+R$. Moreover, $S$ height is $z_{s}=7 \mathrm{~cm}$. Finally, we have to say that circular cylinder UTD scattering is only valid if $k R \geq 2$ [13], i.e. $f \geq 2.73 \mathrm{GHz}$ for the presented comparison: that is why only results for frequencies greater than $3 \mathrm{GHz}$ are presented.

First, results for $\theta=90^{\circ}$ (2D configuration) are given, for $f=4,5 \mathrm{GHz}$ and $d=7 \mathrm{~cm}$, by Figures 4 (a) and 4 (b) for polarizations $\theta$ and $\phi$ respectively. Because the used frequency is greater than $3 \mathrm{GHz}$, we used the

dielectric properties given by [7] for the "Muscle" model, i.e. $\epsilon_{r}=\epsilon_{r}^{\prime}=48.2$ 


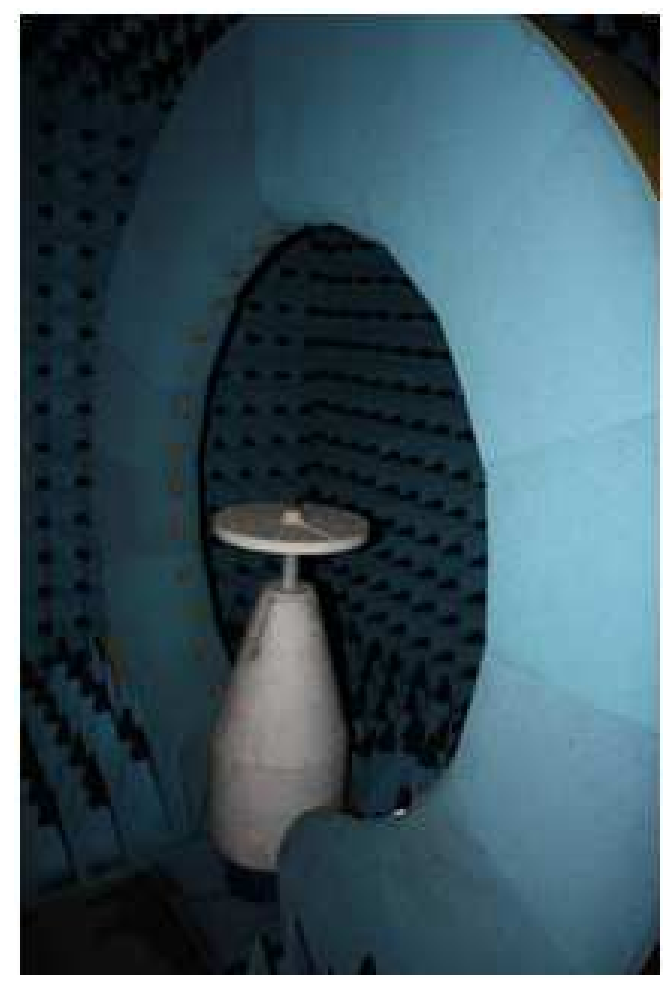

(a) IETR Anechoic Chamber

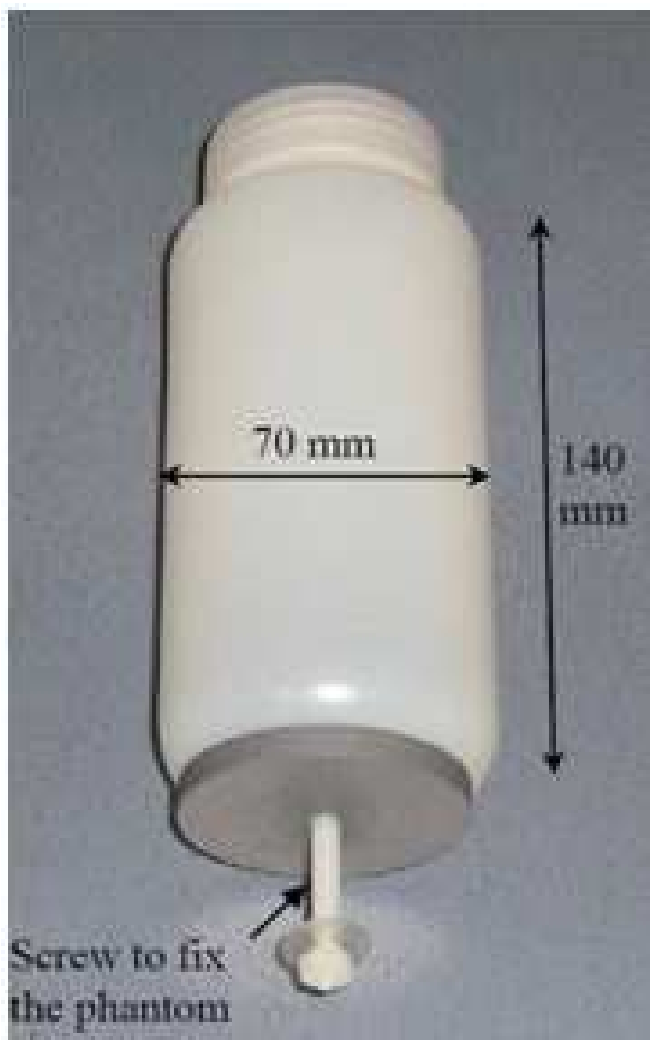

(b) Phantom Dimensions

Figure 2: Measurement Configuration 


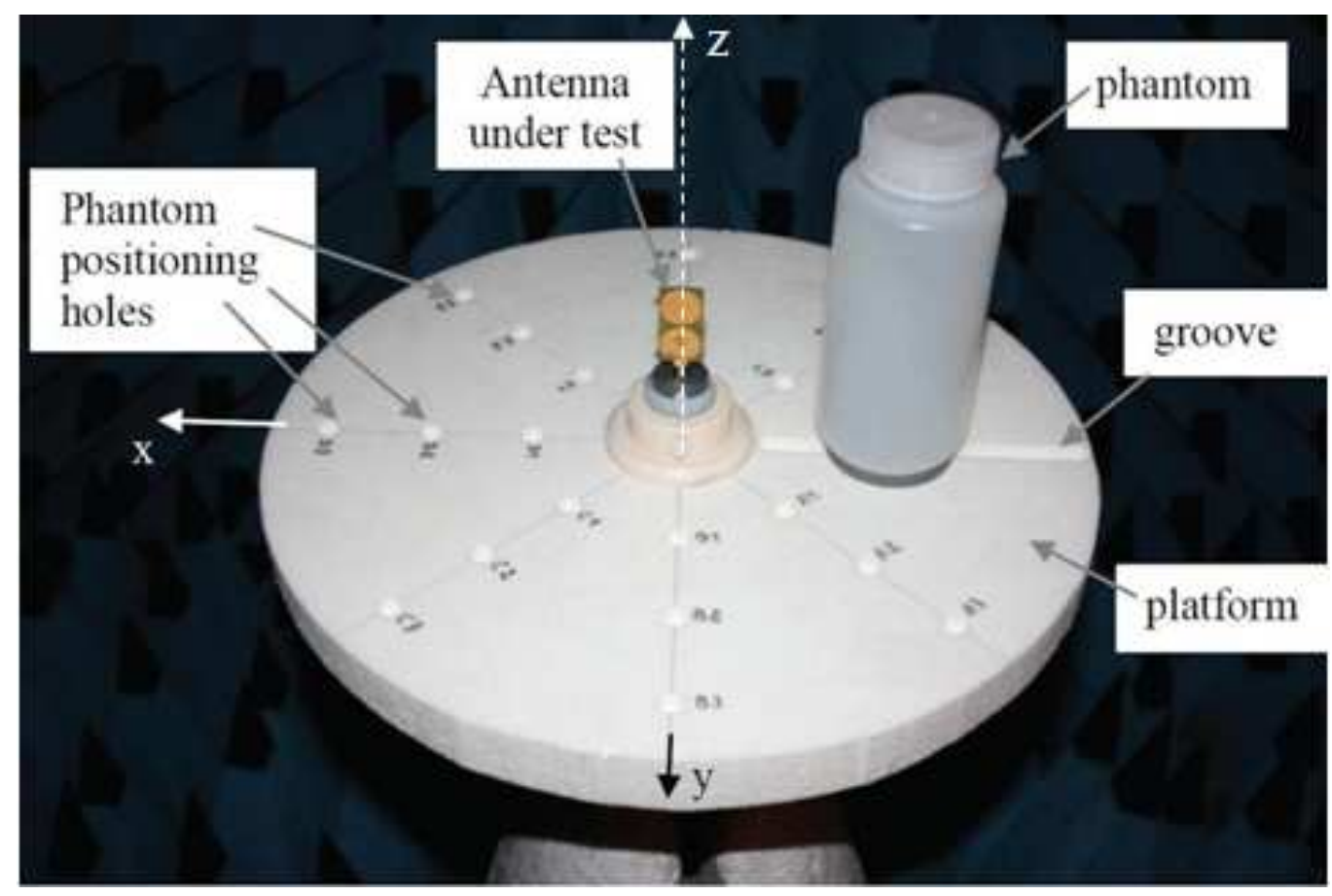

Figure 3: Measurement Coordinates System

and $\sigma=4.7 \mathrm{~S} / \mathrm{m}$. We can notice a very good agreement between simulations and measurements, especially for polarization $\theta$, for which maxima and minima occurs for the same values of $\phi$. The model also predicts a maximum at $\phi=180^{\circ}$ for $\operatorname{NLOS}\left(\phi \in\left[162^{\circ} ; 198^{\circ}\right]\right)$, due to constructive interference between rays 1 and 2 . For polarization $\phi$, the shape of the 2 curves are globally the same with little more variations with the propagation model. We can make the same conclusions for all the other possible scenarios with a minimum usable frequency of $1.5 \mathrm{GHz}$ instead of $2.73 \mathrm{GHz}$ calculated above. Under this minimum frequency, the model results move away from the measurements particularly for the smallest distance $(d=3 \mathrm{~cm})$ for which $S$ seems too close to the cylinder regarding the used wavelength. Moreover, we have to notice that results for polarization $\phi$ are not crucial: because of the used antenna [17], the emitted electric field is necessarily weaker for polarization $\phi$ and close to noise level. So, we will now only focus on polarization $\theta$.

Secondly, for $\theta \neq 90^{\circ}$ (3D configuration), we achieve the same conclusions 


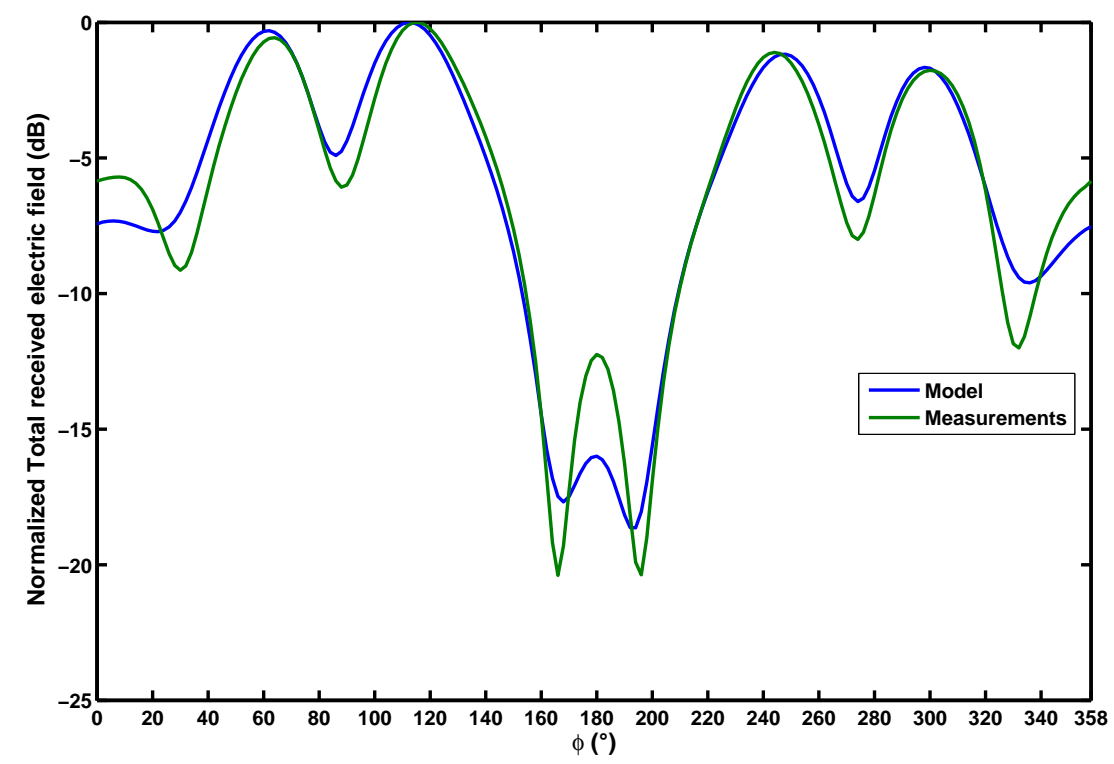

(a) Polarization $\theta$

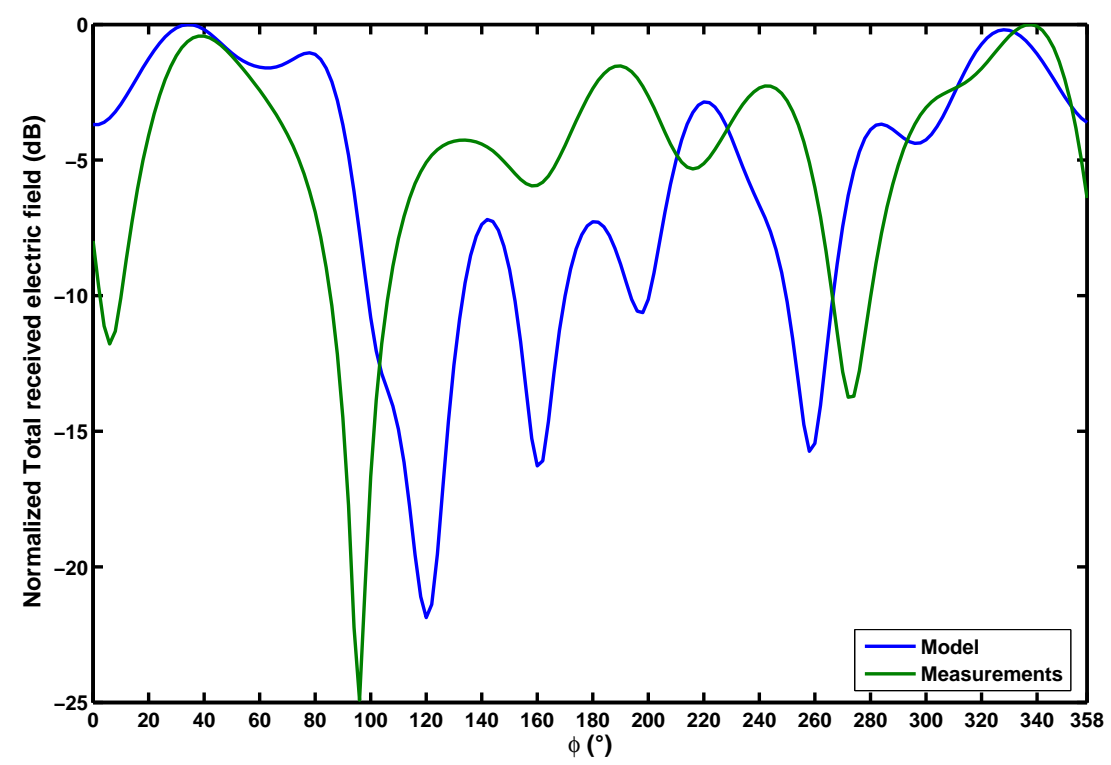

(b) Polarization $\phi$

Figure 4: Comparison for $\theta=90^{\circ}, d=7 \mathrm{~cm}$ and $f=4.5 \mathrm{GHz}$. 
as for 2D configuration when 4 rays are found for LOS and 2 rays are found for NLOS. So we only focus in this paper on the cases for which not all the rays are found. For instance, Fig. 5 gives the results for polarization $\theta$ with $d=13 \mathrm{~cm}$ and $f=3 \mathrm{GHz}$. For $\theta=70^{\circ}$ (Fig. 5 (a)), diffracted rays are only found for $\phi \in\left[142^{\circ} ; 218^{\circ}\right]$ but model results are quite close to the measurements: so we can say that, if the forgotted diffracted rays maybe exist for the measurements, they are so weak that they have no influence on the total received field. For $\theta=64^{\circ}$ (Fig. $5(\mathrm{~b})$ ), the model does not find diffracted rays. Reflected rays are not found for $\phi \in\left[108^{\circ} ; 252^{\circ}\right]$ because of the finite length of the 3D cylinder. And the model predicts no field in the NLOS region, i.e. for $\phi \in\left[170^{\circ} ; 190^{\circ}\right]$, while a received field is measured. This case highlights the fact that the rays diffracted by the bottom and top disks of the cylinder have to be taken into account if we consider a canonical scenario, especially for $\phi \in\left[108^{\circ} ; 252^{\circ}\right]$ that is the $\phi$ range for which model results and measurements are quite different. For $\theta=60^{\circ}$ (Fig. 5 (c)), $S$ and $P$ are always in $L O S$. The model only finds the direct ray because the raytracing finds a reflection point that is always off the cylinder. But we can notice a measurements oscillatory behavior implying interference between, at least, 2 rays: we notice, once again, that additive rays, diffracted by the cylinder disks, have to be taken into account for a canonical case. Finally, for $\theta=6^{\circ}$ (Fig. $5(\mathrm{~d})$ ), the model only predicts a direct ray but, this time, its results are quite close to the measurements: for this case, the influence of the "disk-diffracted" rays seems less problematic.

\section{Conclusion}

A 3D deterministic propagation model, based on ray-tracing and UTD "Scattering" case, has been described and validated for the particular case of a 3D single dielectric circular cylinder of finite length. The developed model originalities are its deterministic nature and its ability to take into account not only the cylinder dielectric nature but also any antenna pattern including NFC (Near-Field Chamber) measured patterns. We have also highlighted the fact that the top and bottom disks cylinder diffracted fields have an influence on the total received field for the presented canonical problem. However, this issue does not exist in a WBAN context.

Now, the model can be improved for the more general case of an elliptic cylinder. But 2 other scenarios could also be interesting for WBAN: the UTD 


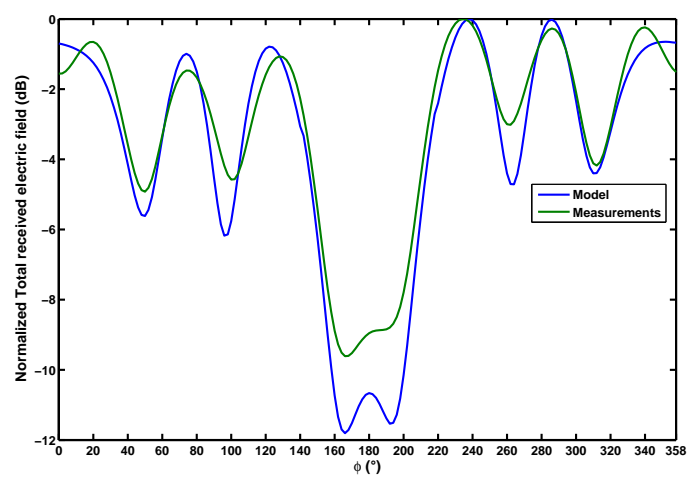

(a) $\theta=70^{\circ}$

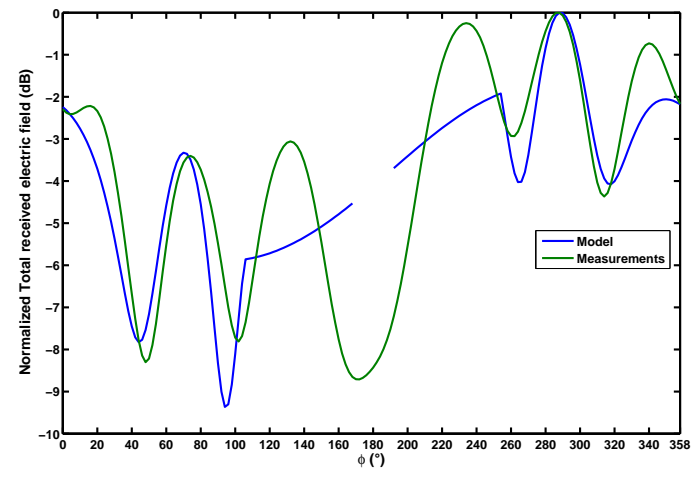

(b) $\theta=64^{\circ}$

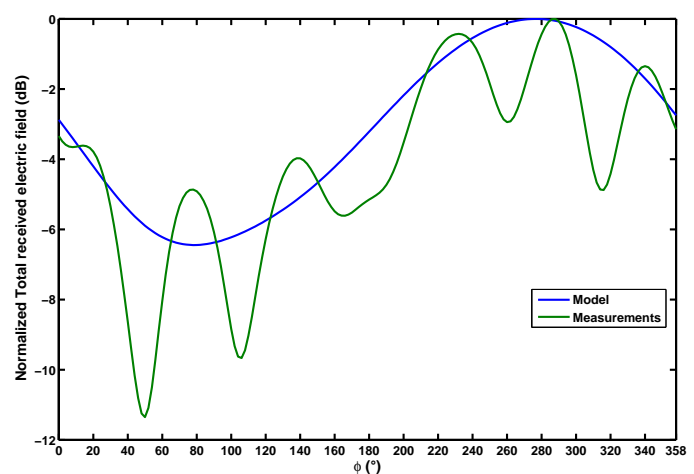

(c) $\theta=60^{\circ}$

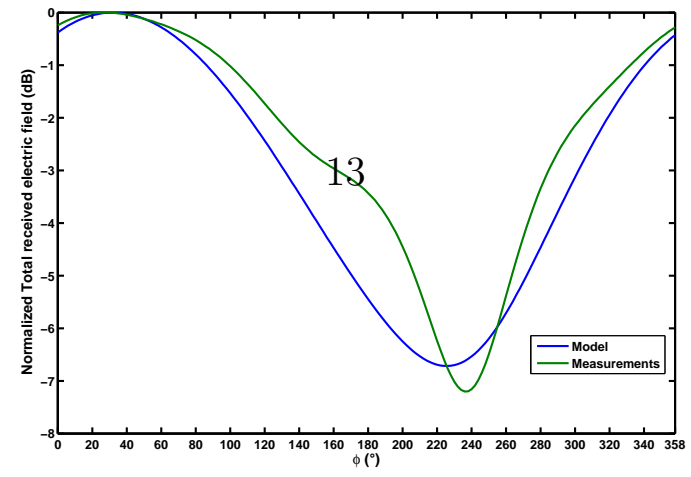

(d) $\theta=6^{\circ}$

Figure 5: Polarization $\theta$ with $d=13 \mathrm{~cm}$ and $f=3 \mathrm{GHz}$. 
"Radiation" scenario (with the Source (resp. the Receiver) on the cylinder and the Receiver (resp. the Source) off the cylinder) and the UTD "Coupling" scenario (when both Source and Receiver are on the cylinder). These scenarios imply the computation of new integrals, different from the Pekeris function of equation (14).

Then, we can also study the possibility of adapting the model for the scenario of several cylinders in order to really model the human body. After, we can imagine a dynamic model taking into account the human body motion. Then we could imagine a scenario with several persons...

This model should also be optimized in term of computation time if it has to be implemented into more complex WBAN propagation models. For this, the model could take advantage of spherical harmonics [1] [18] for antennas pattern description.

\section{Acknowledgment}

This work has been carried out in the frame of the CORMORAN project, which is funded by the French National Research Agency (ANR) under the contract number ANR-11-INFR-010.

\section{References}

[1] M. Mhedhbi, S. Avrillon, B. Uguen, M. Pigeon, R. D'Errico and P. Pasquero, D2.1 - On-Body Antennas Characterization and Exploitable Radiation Properties", Technical Report, CORMORAN (ANR 11-INFR-010) Project, 57 p, October 31, 2012.

[2] R. D'Errico, L. Liu, P. De Doncker, L. Ouvry and C. Oestges, Dynamic Channel Modeling at 2.4 GHz for On-Body Area Networks, Advances in Electronics and Telecommunications, vol. 2, no. 4, pp. 18-27, December 2011.

[3] S. L. Cotton, W. G. Scanlon and B. K. Madahar, Simulation of Millimetre-Wave Channels for Short-Range Body to Body Communications, 2010 Proceedings of the Fourth European Conference on Antennas and Propgation (EuCAP 2010), 5 p., 12-16 April 2010. 
[4] Y. Zhao, Y. Hao, A. Alomainy and C. Parini, UWB On-Body Radio Channel Modeling using Ray Theory and Subband FDTD Method, IEEE Trans. on Microwave Theory and Techniques, vol. 54, no. 4, pp. 18271835, April 2006.

[5] M. Ghaddar, L. Talbi, T. A. Denidni and A. Sebak, A Conducting Cylinder for Modelling Human Body Presence in Indoor Propagation Channel, IEEE Trans. on Antennas and Propagation, vol. 58, no. 2, pp. 503-514, February 2010.

[6] A. A. Goulianos, T. C. Brown, B. G. Evans and S. Stavrou, Wideband Power Modeling and Time Dispersion Analysis for UWB Indoor Off-Body Communications, IEEE Trans. on Antennas and Propagation, vol. 57, no. 7, pp. 2162-2171, July 2009.

[7] G. Koutitas, Multiple Human Effects in Body Area Networks, IEEE Antennas and Wireless Propagation Letters, vol. 9, pp. 938-941, 2010.

[8] R. Chandra and A. J. Johansson, Analytical On-Body Propagation Models for Channel around the Body based on Attenuation of Creeping Waves over an Elliptical Surface, IC1004 TD(13)06053, Malaga, Spain, 6-8 February 2013.

[9] A. Fort, F. Keshmiri, G. R. Crusats, C. Craeye and C. Oestges, A Body Area Propagation Model derived from fundamental Principles: Analytical Analysis and Comparison with Measurements, IEEE Trans. on Antennas and Propagation, vol. 57, no. 7, pp. 2162-2171, July 2009.

[10] D. A. McNamara, C. W. I. Pistorius and J. A. G. Malherbe, Introduction to the Uniform Geometrical Theory of Diffraction, Artech House, 1990.

[11] P. H. Pathak, W. D. Burnside and R. J. Marhefka, A Uniform GTD Analysis of the Diffraction of Electromagnetic Waves by a Smooth Convex Surface, IEEE Trans. on Antennas and Propagation, vol. AP-28, no. 5, pp. 631-642, September 1980.

[12] P. E. Hussar, A Uniform GTD Treatment of Surface Diffraction by Impedance and Coated Cylinders, IEEE Trans. on Antennas and Propagation, vol. 46, no. 7, pp. 998-1008, July 1998. 
[13] H. J. F. G. Govaerts, Electromagnetic Wave Scattering by a Circular Cylinder of Arbitrary Radius, Report of Graduation Work, performed October 1992 - August 1993, 62 p.

[14] W. V. T. Rusch, L. R. W. Welch and G. E. Mires, Observation-PointDependent Blocking Shadows on a Reflector Antenna, IEEE Trans. on Antennas and Propagation, vol. 37, no. 6, pp. 690-697, June 1989.

[15] E. Plouhinec, Etude et Extension de Modèles de Prédiction de la Propagation. Elaboration d'un Serveur Expert Multi-Modèles, PhD Dissertation D 00-13, INSA de Rennes, December 2000.

[16] M. Mhedhbi, Requirements for UWB Antenna Electromagnetic Simulations, CORMORAN Working Document, October 23, 2012.

[17] E. Guéguen, F. Thudor and P. Chambelin, A Low Cost UWB Printed Dipole Antenna with High Performances, 2005 IEEE International Conference on Ultra-Wideband (ICU 2005), pp. 88-92, 5-8 September 2005.

[18] R. Burghelea, S. Avrillon and B. Uguen, Ultra-compact Parametric Modelling of three-dimensional Antenna Radiation Pattern for Impulse Radio Ultra-Wideband Ray-tracing, IET Microwaves, Antennas and Propagation, vol. 6, iss. 11, pp. 1251-1258, 2012. 